\title{
Drug-induced interstitial lung disease in the
} treatment of malignant lymphoma as a potential diagnostic marker: a comparison of serum Krebs von Lungen- 6 and thymus and activation-regulated chemokine/CC chemokine ligand 17

This article was published in the following Dove Press journal: Therapeutics and Clinical Risk Management

\section{Hiromichi Yamane \\ Nobuaki Ochi \\ Yasunari Nagasaki \\ Tomoko Yamagishi \\ Yoshihiro Honda \\ Nozomu Nakagawa \\ Masami Takeyama \\ Hidekazu Nakanishi \\ Nagio Takigawa}

Department of General Internal Medicine 4, Kawasaki Medical School, Okayama, Japan
Correspondence: Hiromichi Yamane Department of General Internal Medicine 4, Kawasaki Medical

School, 2-I-80, Nakasange, Kita-ku,

Okayama 700-8505, Japan

$\mathrm{Tel}+81862252111$

Fax +81862328343

Email hiromichi.ya@gmail.com
Purpose: Cure-oriented treatment of malignant lymphoma (ML) is possible even in an advanced stage; however, the progression of drug-induced interstitial lung disease (DILD) sometimes accounts for poor clinical outcomes. This study aims to assess the incidence and clinical characteristics of DILD among patients with ML and compares the serum level of Krebs von den Lungen-6 (KL-6) with that of circulating thymus and activation-regulated chemokine (TARC)/ CC chemokine ligand 17 (CCL17) as a diagnostic biomarker for DILD.

Patients and methods: Between July 2011 and August 2016, we enrolled 36 patients with ML who were undergoing systemic chemotherapy at our hospital. Then, we evaluated the serum concentration of KL- 6 and TARC/CCL17 by a sandwich-type electrochemiluminescence immunoassay and enzyme-linked immunosorbent assay, respectively.

Results: DILD developed in $22.2 \%$ of patients with ML. All patients recovered immediately after the discontinuation of causative drug and/or glucocorticoid therapy. Although the sensitivity of both TARC/CCL17 and KL-6 was almost equal, the mean concentration of serum KL-6 after the progression of interstitial lung disease was significantly higher than that before progression.

Conclusion: DILD developed in patients who were treated with first-line rituximab combined regimen. Remarkably, TARC/CCL17 and KL-6 seemed approximately equal as a predictive biomarkers for DILD; however, KL-6 was more specific than TARC/CCL17.

Keywords: malignant lymphoma, drug-induced interstitial lung disease, KL-6, TARC/CCL17, biomarker

\section{Introduction}

Most anticancer drugs have the possibility to cause pulmonary adverse effects in the interstitial tissue of the patient's lung. Interstitial lung disease (ILD) is not exceptional and ranges from trivial hypersensitivity reactions to irreversible lung fibrosis and life-threatening acute respiratory distress syndrome. ${ }^{1}$ Although the clinical patterns of drug-induced ILD (DILD) differ based on patients' illness and drug-related factors, ${ }^{2}$ the mainstay of therapeutic intervention is first to diagnose and eliminate the causative chemotherapeutic drugs as soon as possible. Besides our knowledge regarding the risk factors of DILD, the establishment of reasonable early diagnostic methods is crucial because more comprehensive insights into the underlying mechanisms in the development of DILD are yet to be clarified. ${ }^{3}$ Although chest high-resolution CT (HRCT) 
is a potent diagnostic method for detecting ILD, the risk of developing radiation-induced adverse events, including carcinogenesis following CT screening is a current topic for candidates all over the world. ${ }^{4}$ Hence, the establishment of useful diagnostic markers in the serum is imperative.

Kohno et $\mathrm{al}^{5}$ have extensively investigated Krebs von den Lungen-6 (KL-6), human MUC1 mucin. Reportedly, KL-6 is considered as type II pneumocyte biomarker with clear correlation with clinical manifestations of idiopathic pulmonary fibrosis (IPF) and reflects the disease condition of various ILDs. ${ }^{6,7}$

Thymus and activation-regulated chemokine (TARC)/CC chemokine ligand 17 (CCL17), a functional ligand for CC chemokine receptor type 4 , is reportedly elevated in the bronchoalveolar lavage fluids of the patient who has acute eosinophilic pneumonia (AEP). ${ }^{8}$ Furthermore, TARC/CCL17 has been proven to be predominantly localized to the pulmonary epithelial cells in a mouse model of bleomycin-induced fibrosis and patients with IPF. ${ }^{9}$ In addition, studies have reported that TARC/CCL17 in sera are increased and correlated with the disease status of allergic diseases, such as atopic dermatitis, bronchial asthma, and AEP, and lymphoid malignancies, including classical Hodgkin's lymphoma and mycosis fungoides. ${ }^{10,11}$ It is assumed that circulating TARC/CCL17 level reflects in situ immunological reactions induced by Th2 cytokines in various diseases. ${ }^{12}$ In addition, we have previously reported that TARC/CCL17 is a strong candidate diagnostic marker for ILD in patients with malignant tumors. ${ }^{12}$

Since serum levels of TARC/CCL17 are considered to be associated with the disease activity of lymphoid malignancies, this study aims to retrospectively elucidate the probability and characteristics of DILD among patients with malignant lymphoma (ML) and, then, compare the serum level of KL-6 with that of TARC/CCL17 as a diagnostic biomarker for DILD.

\section{Materials and methods Study population}

We enrolled 36 patients who were diagnosed as ML (age: 33-88 years; 22 males and 14 females; mean \pm SD: 69.4 \pm 11.9 years) between July 2011 and August 2016 who were hospitalized in the Department of General Internal Medicine 4, Kawasaki Medical School General Medical Center (Okayama, Japan). The inclusion criteria in this study were as follows: 1) pathological diagnosis of ML and 2) undergoing systemic chemotherapy. Of note, the inclusion criteria were not based on age, gender, treatment line, or smoking habits.
ILD was diagnosed on the basis of disease history, physical examinations, studies of pulmonary function, arterial blood gas analysis, and chest HRCT. ${ }^{13}$ However, the radiological diagnosis on the chest HRCT reportedly reflected 4 major pathological subtypes of ILD, diffuse alveolar damage (DAD), bronchiolitis obliterans with organizing pneumonia or eosinophilic pneumonia (BOOP/EP), and hypersensitivity pneumonitis (HP). ${ }^{7,13,14}$ Notably, chest CT scans of all enrolled patients at an initial diagnosis of ML were reviewed by board-certified members of the Japan Radiological Society and Japanese Respiratory Society. Patients who developed DILD were reviewed again and further classified into these 4 categories by these board-certified members. We analyzed peripheral venous blood samples, which were collected from patients upon their initial admission and were stored at $-80^{\circ} \mathrm{C}$ after the progression of ILD until use, in a blinded manner with regard to patients' clinical status. This study conformed to the Declaration of Helsinki (1975) and was approved by the Kawasaki Medical University Ethics Committee (number 1605). We obtained written informed consent from all participants before initiating treatment of ML.

\section{Measurement of serum KL-6 concentration}

We measured the serum concentration of KL-6 with outsourcing to external laboratory using a sandwich-type electrochemiluminescence immunoassay (BML, Inc., Tokyo, Japan). The cutoff level $(500 \mathrm{U} / \mathrm{mL})$ was set as the level that resulted in the optimal diagnostic accuracy, as described previously for healthy volunteers (mean \pm SD for healthy volunteers). ${ }^{12}$

\section{Measurement of serum TARC/CCLI7 concentrations}

We used commercially available enzyme-linked immunosorbent assay (ELISA) kit (ITSI-Biosciences, Johnstown, PA, USA) to evaluate the concentration of TARC/CCL17 according to the manufacturer's guidance for the cryopreserved serum. The cutoff level $(450 \mathrm{pg} / \mathrm{mL})$ was set as the level that resulted in the optimal diagnostic accuracy, as described previously for healthy volunteers (mean \pm 2 SD for healthy volunteers). ${ }^{12,15}$

\section{Statistical analysis}

We used Student's $t$-test or the Mann-Whitney $U$-test, if applicable, to assess differences in the levels of serum markers between the subject groups. In addition, the chi-squared test for the goodness of fit or the Fisher's exact probability test was used to assess positive quantitative differences. 
The significance of the positive ratio of KL-6 and TARC/ CCL17 was tested using the chi-squared test. Furthermore, receiver operating characteristic (ROC) curves of KL-6 and TARC/CCL17 were performed using the STATA software (Light Stone Corp., Tokyo, Japan). In this study, all $P$-values corresponded to 2 -sided tests and the significance was set at $P<0.05$.

\section{Results}

\section{Characteristics of the study population}

Table 1 presents the characteristics of all enrolled patients in this study. Except for 1 patient with Welder's pneumoconiosis, no patient had a history of ILD. In our study population, the common histological diagnoses were diffuse large B-cell lymphoma (DLBCL; $n=17)$, follicular lymphoma $(n=11)$, mantle cell lymphoma $(n=3)$, and others $(n=5$; Figure $1 A)$. In addition, $44.4 \%$ (16 of 36) of patients were current or ex-smokers, and $16.6 \%$ (6 of 36) had a habit of heavy smoking (Brinkman Index over 600). DILD was progressed in 8 of all patients $(22.2 \%$; 95\% CI: $10.1 \%-39.2 \%)$, including a patient with a history of Welder's pneumoconiosis, and all causative treatments comprised "doxorubicin, cyclophosphamide, vincristine, prednisone (CHOP)-like regimen" as the first-line chemotherapy (rituximab, cyclophosphamide, doxorubicin, vincristine, and prednisolone [R-CHOP]: 4; rituximab, tetrahydropyranyl-adriamycin, cyclophosphamide, vincristine, and prednisolone [R-THP-COP]: 4; Figure 1B). Figure 1 shows the detailed breakdown of diseases and the adapted initial treatments.

\section{Radiological diagnosis and clinical outcome of patients with DILD}

Figure 2 shows the radiological subtype of patients with DILD and their overall survival. Among 8 patients with ML in whom DILD progressed, 6 were diagnosed with HP, and 1 with DAD, and another with AEP (Figure 3A). In this

Table I Patient characteristics $(n=36)$

\begin{tabular}{lll}
\hline Characteristics & & \\
\hline Age, mean \pm SD, range & $69.4 \pm 11.9$ & $33-88$ \\
Male, n (\%) & 22 & 61.10 \\
Smoking, n (\%) & 16 & 44.40 \\
Heavy smoking (BI over 600), n (\%) & 6 & 16.60 \\
Malignant tumors, n (\%) & & \\
$\quad$ Past history & 9 & 25.00 \\
$\quad$ Family history & 12 & 33.30 \\
Development of drug induced ILD, n (\%) & 8 & 22.20 \\
History of prior ILD, n (\%) & 1 & 2.8 \\
Ethnicity (Japanese), n (\%) & 36 & 100 \\
\hline
\end{tabular}

Abbreviations: $\mathrm{BI}$, Brinkman Index; ILD, interstitial lung disease. study, all DILDs progressed in the periods of the first-line treatment and the causative regimens were "CHOP-like" regimen. Since these patients with DILD recovered after the withdrawal of chemotherapeutic regimen and/or steroid administration, the clinical outcome of these patients seemed acceptable (Table 2). In addition, the median survival of patients with DILD was 33.0 months and their 1-year survival rate was $87.5 \%$ and $58.3 \%$, respectively; these data seemed not to be inferior compared with previous reports of the clinical outcome of aggressive lymphoma (Figure 3B). ${ }^{16}$

\section{Serum levels of circulating KL-6 and TARC/CCLI7}

When the written informed consent from the enrolled patients measuring each surrogate markers using preserved serum before initial treatment was provided, the examination was carried out and acquired data were analyzed. In some patients, we could obtain a value of TARC/CCL17 and KL-6 from their medical record as clinical information. We performed the data acquisition from medical record in those cases. In all acquired data, we judged the data above the standard value were positive, and the data within the standard value were judged as negative. Then, the sensitivity, specificity, and prevalence rate of each marker were calculated by the DILD emergence.

Because the method for measuring KL-6 adopted in our hospital was a sandwich-type electrochemiluminescence immunoassay and that of TARC/CCL17 was an ELISA, we used the same method in case of surrogate marker examination using preserved serum. Table 3 explains the sensitivity, specificity, and prevalence rates associated with the cutoff levels of each marker in this study. We used previously reported values of KL-6 and TARC/CCL17 in Japanese healthy volunteers as reference. ${ }^{11,14}$ The results revealed that the sensitivity of both markers to detect DILD was potentially equal. Both markers could act as a weak surrogate marker among almost $50 \%$ of patients with DILD. However, these results were remarkably lower than our initial expectation. Contrary to the results of sensitivity, the specificity of KL-6 was more likely to be higher than those of TARC/CCL17, although the difference was not statistically significant $(P=0.465)$.

In addition, the ROC curve of each marker revealed equal efficiency as a surrogate marker for DILD. The values of the area under the ROC curve of KL- 6 and TARC/CCL17 were 0.73 and 0.77 , respectively, and we observed no remarkable difference between these 2 markers ( $P=0.974$; Figure 4).

Figure 2 shows circulating TARC/CCL17 and KL-6 levels on admission and after progression of DILD. The comparison of TARC/CCL17 levels is shown in Figure 2. 
A

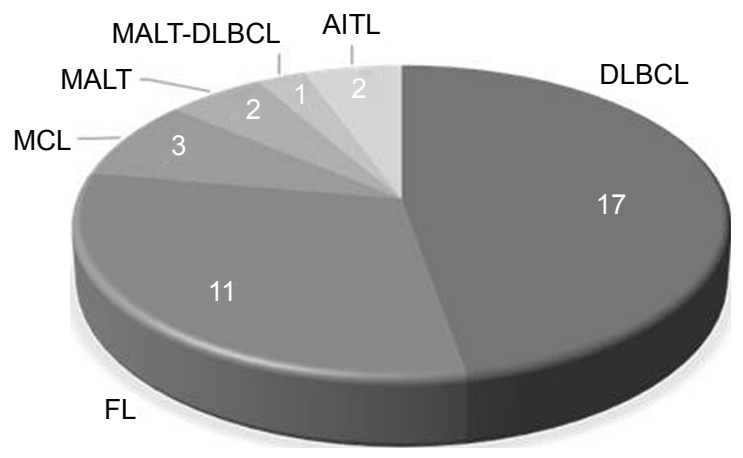

B

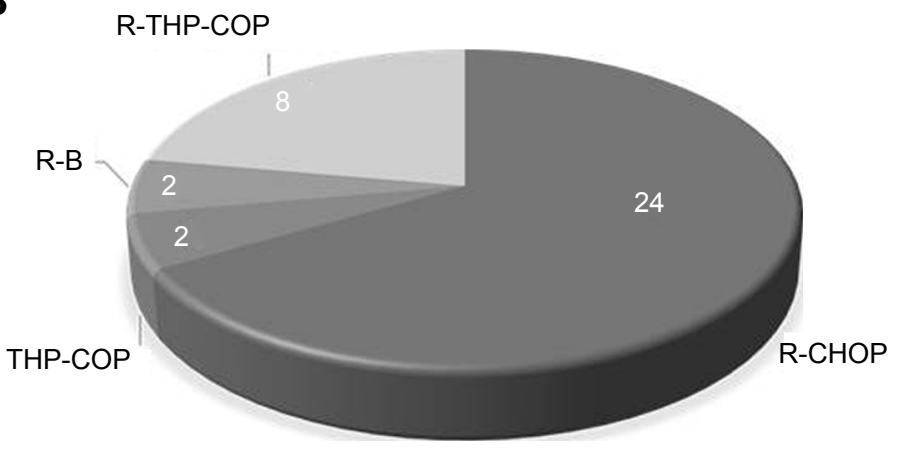

Figure I Demographic characteristics of the study population.

Notes: (A) Pathological diagnosis. The common histological diagnoses were DLBCL $(n=17), F L(n=I I), M C L(n=3)$, and others ( $n=5)$. Others included AITL, extranodal marginal-zone B-cell lymphoma MALT type, and DLBCL transformed from extranodal marginal-zone B-cell lymphoma MALT type (MALT-DLBCL). (B) Regimens of the firstline treatment. In this study, 34 of 36 patients with ML received the so-called "CHOP-like" regimen as the first-line chemotherapy. Drug-induced ILD progressed in periods of first-line treatment, and all causative regimens were "CHOP-like."

Abbreviations: AITL, angioimmunoblastic T-cell lymphoma; DLBCL, diffuse large B-cell lymphoma; FL, follicular lymphoma; ILD, interstitial lung disease; MALT, mucosaassociated lymphoid tissue; MCL, mantle cell lymphoma; ML, malignant lymphoma; R-B, rituximab and bendamustine; R-CHOP, rituximab, cyclophosphamide, doxorubicin, vincristine, and prednisolone; R-THP-COP, rituximab, tetrahydropyranyl-adriamycin, cyclophosphamide, vincristine, and prednisolone; THP-COP, tetrahydropyranyladriamycin, cyclophosphamide, vincristine, and prednisolone.

The average concentration of TARC/CCL17 level on admission was $483.8 \pm 705.1 \mathrm{pg} / \mathrm{mL}$ and that after progression of DILD was $746.0 \pm 766.1 \mathrm{pg} / \mathrm{mL}$. We observed no significant difference between 2 groups $(P=0.439)$. Next, we examined circulating KL-6 levels on admission and after progression of DILD. The average concentration of KL-6 level on admission was $338.4 \pm 172.7 \mathrm{U} / \mathrm{mL}$ and that after progression of DILD was $719.9 \pm 493.9 \mathrm{U} / \mathrm{mL}$. The average concentration of KL-6 after progression of DILD was higher than that on admission, and a significant difference was observed between 2 groups $(P=0.0028$; Figure 2B).

\section{Discussion}

DILD is a typical adverse event that poses a critical problem in the management of patients during cancer treatment. Although detailed and accurate epidemiological data about DILD in the treatment of ML have lacked for the last 2 decades, some critical reports have been published in recent years. ${ }^{17-19}$ Summaries of these pivotal reports are as follows: 1) DILD is a life-threatening complication in patients with ML. However, the incidence of DILD was about $2 \%-5 \%$ of patients with B-cell non-Hodgkin lymphoma, primarily including DLBCL. 2) The addition of rituximab to chemotherapy might be identified as one of the significant risk factors of ILD with a trend toward a higher incidence. 3) Glucocorticoid therapy might be a potent strategy for the management of these ILD. The results of this study emphasize that glucocorticoid therapy and immediate elimination of causative agents might be the most critical measure for DILD.

However, the incidence of DILD in this study was about 3- to 4-fold higher than that of the other reports; the reason of this high incidence could be attributed to the diagnostic methods for detecting DILD and the causative chemotherapeutic agents. In our division, we routinely check chest HRCT every 8 weeks to evaluate the efficacy of anticancer agents and detect ILD among patients with ML under chemotherapy (Figure S1). Performing such a diagnostic procedure might 



Figure 2 A comparison of the average concentration of serum TARC/CCLI7 and KL-6 before/after the development of drug-induced ILD.

Notes: (A) TARC/CCLI7; (B) KL-6. Although no significant difference was observed between the average concentration of the serum TARC/CCLI7 level on admission and that after the progression of drug-induced ILD ( $P=0.439)$, the average concentration of serum KL-6 after the progression of ILD was significantly higher than that on admission $(P=0.0028)$.

Abbreviations: ILD, interstitial lung disease; KL-6, Krebs von den Lungen-6; TARC/CCLI7, thymus and activation-regulated chemokine/CC chemokine ligand I7; NS, not significant.

lead to early detection of DILD. In fact, half of the patients (4 of 8) in this study recovered by the discontinuance of an anticancer agent without glucocorticoid therapy. Furthermore, all patients with DILD recovered using glucocorticoid therapy and/or the discontinuance of anticancer agents, indicating that "asymptomatic" patients with these DILD had a good clinical outcome and were detected more than "symptomatic" patients. In addition, all patients with DILD in this study occurred in the "rituximab combined CHOP-like regimen" as the first-line treatment. Of note, the incidences of DILD might differ in each surveillance schedules of DILD and chemotherapeutic methods.

The development of surrogate diagnostic biomarkers is crucial for the early and reliable diagnosis of DILD. KL-6 is believed to be the most reliable serum biomarker for the diagnosis and management of "popular" ILD. ${ }^{20}$ However, we previously revealed that TARC/CCL17 might be more useful than KL-6 as a diagnostic marker in some patients with cancer. Both the sensitivity and specificity of TARC/ CCL17 to detect DILD were higher than those of KL-6 in patients with NSCLC. ${ }^{12}$ These results might be attributed to the following reasons: 1) multitudinous factors, such as smoking status and occupational history, which regulate the immune and inflammatory response have participated in the pathogenesis of ILD. ${ }^{21-25}$ 2) Regardless of the causing agents, the hallmark of DILD is chronic inflammation and deposition of extracellular matrix..$^{26} 3$ ) The phenotype of this chronic inflammation is possibly related to the Th2 phenotype of the cytokine expression. ${ }^{27}$

Besides these hypotheses, we should initiate a clinical study comparing the usefulness of TARC/CCL17, which is one of the most famous Th2 cytokine, and KL-6, which is the most robust candidate biomarker for DILD, among patients with ML because the increase in the serum TARC/CCL17 level was definitely confirmed in proportion to disease progression of ML. In our opinion, TARC/CCL17 was not useful when compared with KL-6 as a biomarker of DILD among patients with ML initially. However, the sensitivity of both markers to detect DILD was potentially equal, and the specificity of serum KL-6 levels tended to be higher than those of TARC/CCL17. Perhaps, multiple factors could participate in these results, for example, smoking status, the 
A

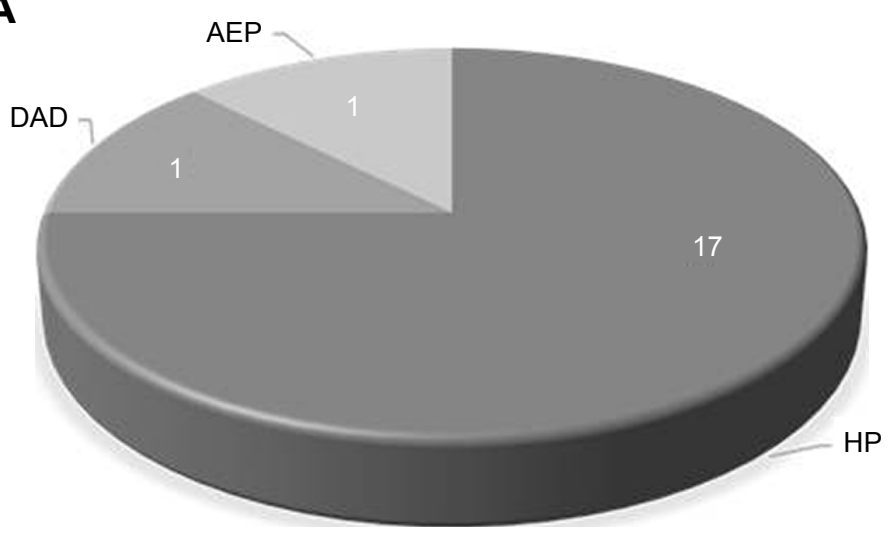

B

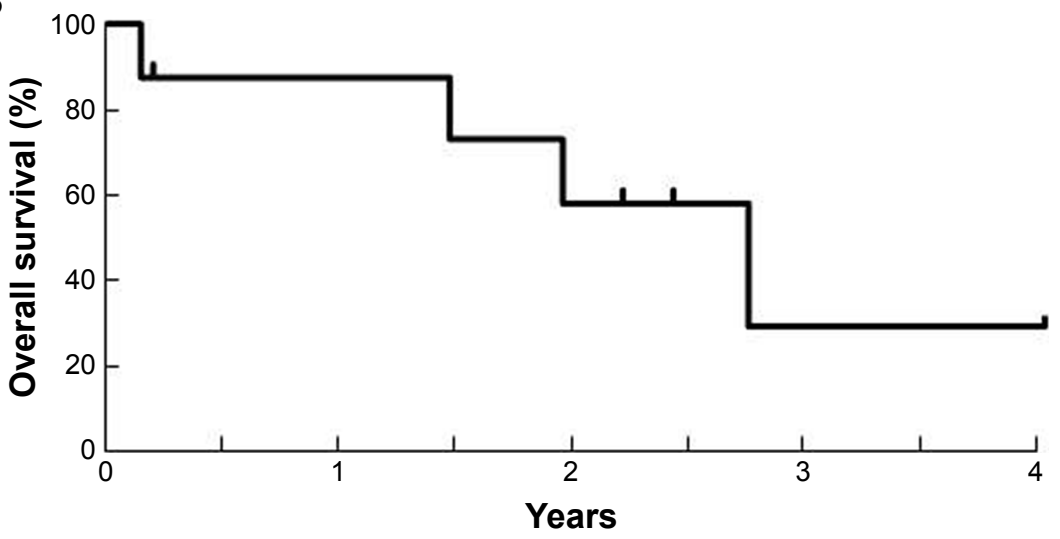

Figure 3 Radiological subtype and the overall survival of DILD.

Notes: (A) A radiological subtype of DILD. Six patients were diagnosed with HP, I with DAD, and I with AEP. (B) The overall survival of patients with DILD. The median survival of patients with DILD was 33.0 months and I- and 2-year survival rates were $87.5 \%$ and $58.3 \%$, respectively.

Abbreviations: AEP, acute eosinophilic pneumonia; DAD, diffuse alveolar damage; DILD, drug-induced interstitial lung disease; HP, hypersensitivity pneumonitis.

background of patients and tumorigenesis, the number of events in our study, and occupational history.

The correlation between the radiological subtype variation and the risk factors of DILD seemed to be even more critical. DAD subtype and AEP subtype were diagnosed in only 1 patient in our study. The serum TARC/CCL17 level in a patient with AEP was elevated over the quadruple of upper normal limit. In addition, the serum KL-6 level was elevated in this case; however, the value of KL-6 was 1.6 times the upper normal limit (KL-6: $833 \mathrm{U} / \mathrm{mL})$.

Both serum TARC/CCL17 and KL-6 levels were slightly elevated than the upper normal limit in a patient with DAD

Table 2 Summary of clinical outcome in the patients with drug-induced ILD

\begin{tabular}{|c|c|c|c|c|c|c|}
\hline $\begin{array}{l}\text { Pt ID/age } \\
\text { (years) }\end{array}$ & $\begin{array}{l}\text { Radiological } \\
\text { subtype of drug- } \\
\text { induced ILD }\end{array}$ & $\begin{array}{l}\text { Treatment } \\
\text { of ILD }\end{array}$ & Recovery & $\begin{array}{l}\text { Dead/ } \\
\text { alive }\end{array}$ & Reason of death & $\begin{array}{l}\text { Survival } \\
\text { (days) }\end{array}$ \\
\hline $\mathrm{Ptl} / 66$ & DAD & PSL $1.0 / \mathrm{kg}$ & Yes & Alive & NR & 1,453 \\
\hline Pt2/88 & $\mathrm{HP}$ & Drug off & Yes & Dead & Heat stroke & 994 \\
\hline $\mathrm{Pt} 3 / 79$ & $\mathrm{HP}$ & PSL 0.5/kg & Yes & Dead & Disease progression & 710 \\
\hline Pt4/76 & $\mathrm{HP}$ & None & Yes & Dead & Pneumonia & 531 \\
\hline$P t 5 / 65$ & AEP & PSL $0.5 / \mathrm{kg}$ & Yes & Dead & Hepatic failure & 53 \\
\hline Pt6/66 & HP & PSL $1.0 / \mathrm{kg}$ & Yes & Alive & NR & 889 \\
\hline Pt7/75 & $\mathrm{HP}$ & Drug off & Yes & Alive & NR & 801 \\
\hline Pt8/76 & $\mathrm{HP}$ & None & Yes & Alive & NR & 71 \\
\hline
\end{tabular}

Abbreviations: AEP, acute eosinophilic pneumonia; DAD, diffuse alveolar damage; HP, hypersensitivity pneumonitis; ILD, interstitial lung disease; NR, not reported; PSL, prednisolone; Pt, patient. 
Table 3 Diagnostic value of measurement of circulating TARC/ CCLI7 and KL-6

\begin{tabular}{|c|c|c|c|}
\hline $\begin{array}{l}\text { Sensitivityl } \\
\text { specificityl } \\
\text { prevalence }\end{array}$ & TARC/CCLI7 & KL-6 & $\begin{array}{l}\text { Chi-squared } \\
\text { test }\end{array}$ \\
\hline Sensitivity & $50.0 \%(4 / 8)$ & $50.00 \%(4 / 8)$ & \\
\hline $\begin{array}{l}\text { From the serum } \\
\text { before ILD }\end{array}$ & $20.00 \%(1 / 5)$ & $16.70 \%(1 / 6)$ & \\
\hline $\begin{array}{l}\text { From the serum } \\
\text { after ILD }\end{array}$ & $50.0 \%(3 / 6)$ & $57.10 \%(4 / 7)$ & \\
\hline Specificity & $80.0 \%(12 / 15)$ & $94.40 \%(17 / 18)$ & NS $(P=0.465)$ \\
\hline Prevalence rate & $34.8 \%(8 / 23)$ & $30.80 \%(8 / 26)$ & \\
\hline
\end{tabular}

Abbreviations: ILD, interstitial lung disease; KL-6, Krebs von den Lungen-6 TARC/CCLI7, thymus and activation-regulated chemokine/CC chemokine ligand 17; NS, not significant.

(TARC/CCL17, $521 \mathrm{pg} / \mathrm{mL}$; KL-6, $722 \mathrm{U} / \mathrm{mL}$ ). Since this patient was also a heavy smoker (BI: 690), there might be some relationship between smoking status and radiological subtype variation. Regarding these phenomena, Ohnishi et al reported that the average serum KL-6 level among patients with DAD was higher than that of $\mathrm{HP}$ and $\mathrm{BOOP} / \mathrm{AEP},{ }^{28}$ and Ritter et al suggested that smoking caused the elevation in the TARC/CCL17 level both in the serum and bronchoalveolar lavage fluid. ${ }^{29}$

This study has some limitations. First, owing to its small sample size and a potential patient selection bias, confirmation of the present results by an extensive study is warranted to ensure the generalizability of our data. Second,
DILD was diagnosed in only 8 patients. Whether sequential measurement of the circulating KL-6 level is useful for the early detection of DILD in the treatment of ML should be clarified in a prospective study.

We would propose a prospective large scale observational study to confirm the incidence and clinical characteristics of DILD among the patients with non-Hodgkin lymphoma (NHL) first. Then, we would confirm the difference of these results between the ethnicities and treatment regimens.

\section{Conclusion}

DILD developed in about $20 \%$ of patients with NHL in our investigation. The clinical outcome of patients with DILD was acceptable. Contrary to our expectations, TARC/ CCL17 and KL-6 seemed to be approximately equal as a biomarker for DILD among patients with NHL. The specificity of KL-6 as a biomarker for DILD tended to be superior to that of TARC/CCL17 and the mean concentration of serum KL-6 after the progression of DILD was significantly higher than that before progression. Based on the results of this study, KL-6 seemed to be a better biomarker for DILD in NHL treatment. Nevertheless, confirmation of the results by a prospective large-scale study is warranted.

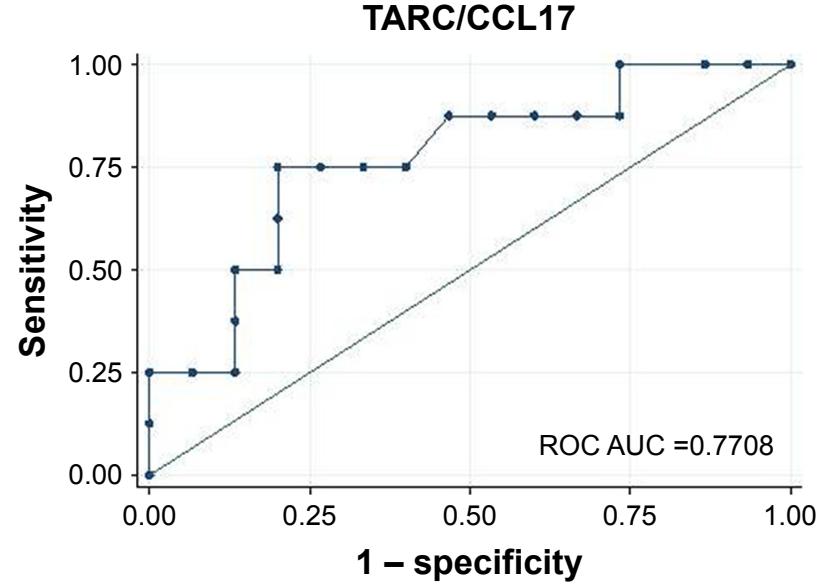

ROC AUC $=0.77$



$\operatorname{ROC} \mathrm{AUC}=0.73$

NS $(P=0.974)$

Figure 4 The ROC curves of TARC/CCLI7 and KL-6 as a surrogate diagnostic marker of DILD. AUC of TARC/CCLI7 and KL-6 detecting DILD proved to be nearly equal.

Abbreviations: AUC, area under the curve; DILD, drug-induced interstitial lung disease; KL-6, Krebs von den Lungen-6; ROC, receiver operating characteristic; TARC/ CCLI7, thymus and activation-regulated chemokine/CC chemokine ligand 17; NS, not significant. 


\section{Acknowledgments}

We thank Professor Katsuya Kato, Department of Radiology, Kawasaki Medical School General Medical Center, for helpful comments and radiological diagnosis of drug-induced ILD. This work was supported, in part, by a Research Support Grant, 15S01242, from Takeda Pharmaceutical Company Limited.

\section{Author contributions}

HY and NT planned this study. TY, NN, and MT measured circulating TARC/CCL17, concentration using ELISA. NO, HY, YN, HN, and YH analyzed the data. HY and NT wrote the paper. All authors contributed toward data analysis, drafting and critically revising the paper, gave final approval of the version to be published, and agree to be accountable for all aspects of the work.

\section{Disclosure}

The authors report no conflicts of interest in this work, including any financial and personal relationships with people or organizations that could inappropriately influence the work.

\section{References}

1. Saito Y, Gemma A. Current status of DILD in molecular targeted therapies. Int J Clin Oncol. 2012;17(6):534-541.

2. Camus $P$, Kudoh S, Ebina M. Interstitial lung disease associated with drug therapy. Br J Cancer. 2004;91 Suppl 2:S18-S23.

3. Hotta K, Kiura K, Takigawa N, et al. Comparison of the incidence and pattern of interstitial lung disease during erlotinib and gefitinib treatment in Japanese Patients with non-small cell lung cancer: the Okayama Lung Cancer Study Group experience. J Thorac Oncol. 2010;5(2): 179-184.

4. Shah DJ, Sachs RK, Wilson DJ. Radiation-induced cancer: a modern view. Br J Radiol. 2012;85(1020):e1166-e1173.

5. Kohno N, Kyoizumi S, Awaya Y, Fukuhara H, Yamakido M, Akiyama M. New serum indicator of interstitial pneumonitis activity. Sialylated carbohydrate antigen KL-6. Chest. 1989;96(1):68-73.

6. Sakamoto K, Taniguchi H, Kondoh Y, et al. Serum KL-6 in fibrotic NSIP: Correlations with physiologic and radiologic parameters. Respir Med. 2010;104(1):127-133.

7. Ohnishi H, Yokoyama A, Kondo K, et al. Comparative study of KL-6, surfactant protein-A, surfactant protein-D, and monocyte chemoattractant protein-1 as serum markers for interstitial lung diseases. $\mathrm{Am} \mathrm{J}$ Respir Crit Care Med. 2002;165(3):378-381.

8. Miyazaki E, Nureki S, Fukami T, et al. Elevated levels of thymus- and activation-regulated chemokine in bronchoalveolar lavage fluid from patients with eosinophilic pneumonia. Am J Respir Crit Care Med. 2002;165(8):1125-1131.

9. Belperio JA, Dy M, Murray L, et al. The role of the Th2 CC chemokine ligand CCL17 in pulmonary fibrosis. J Immunol. 2004;173(7): 4692-4698.

10. Miyazaki E, Nureki S, Ono E, et al. Circulating thymus- and activationregulated chemokine/CCL17 is a useful biomarker for discriminating acute eosinophilic pneumonia from other causes of acute lung injury. Chest. 2007;131(6):1726-1734.
11. Jones K, Vari F, Keane C, et al. Serum CD163 and TARC as disease response biomarkers in classical Hodgkin lymphoma. Clin Cancer Res. 2013;19(3):731-742.

12. Yamane H, Ochi N, Yamagishi T, Honda Y, Takeyama M, Takigawa N. Circulating thymus and activation-regulated chemokine/CC chemokine ligand 17 is a strong candidate diagnostic marker for interstitial lung disease in patients with malignant tumors: a result from a pilot study. Ther Clin Risk Manag. 2015;11:949-959.

13. Travis WD, Costabel U, Hansell DM, et al. An official American Thoracic Society/European Respiratory Society statement: Update of the international multidisciplinary classification of the idiopathic interstitial pneumonias. Am J Respir Crit Care Med. 2013;188(6): 733-748.

14. Webb WR, Müller NL, Naidich DP. High-Resolution CT of the Lung. 3rd ed. Philadelphia: Lippincott Williams \& Wilkins; 2001.

15. Kakinuma T, Wakugawa M, Nakamura K, Hino H, Matsushima K, Tamaki K. High level of thymus and activation-regulated chemokine in blister fluid and sera of patients with bullous pemphigoid. $\mathrm{Br} J$ Dermatol. 2003;148(2):203-210.

16. International Non-Hodgkin's Lymphoma Prognostic Factors Project. A predictive model for aggressive non-Hodgkin's lymphoma. $N$ Engl J Med. 1993;329(14):987-994.

17. Salmasi G, Li M, Sivabalasundaram V, et al. Incidence of pneumonitis in patients with non-Hodgkin lymphoma receiving chemoimmunotherapy with rituximab. Leuk Lymphoma. 2015;56(6):1659-1664.

18. Liu WP, Wang XP, Zheng W, et al. Incidence, clinical characteristics, and outcome of interstitial pneumonia in patients with lymphoma. Ann Hematol. 2018;97(1):133-139.

19. Huang YC, Liu CJ, Liu CY, et al. Low absolute lymphocyte count and addition of rituximab confer high risk for interstitial pneumonia in patients with diffuse large B-cell lymphoma. Ann Hematol. 2011; 90(10):1145-1151.

20. Ishikawa N, Hattori N, Yokoyama A, Kohno N. Utility of KL-6/MUC1 in the clinical management of interstitial lung diseases. Respir Investig. 2012;50(1):3-13.

21. Fujiwara Y, Kiura K, Toyooka S, et al. Elevated serum level of sialylated glycoprotein KL-6 predicts a poor prognosis in patients with non-small cell lung cancer treated with gefitinib. Lung Cancer. 2008; 59(1):81-87.

22. Kobayashi H, Kanoh S, Motoyoshi K. Serum surfactant protein-A, but not surfactant protein-D or KL-6, can predict preclinical lung damage induced by smoking. Biomarkers. 2008;13(4):385-392.

23. Feghali-Bostwick CA, Tsai CG, Valentine VG, et al. Cellular and humoral autoreactivity in idiopathic pulmonary fibrosis. J Immunol. 2007; 179(4):2592-2599

24. Zuo F, Kaminski N, Eugui E, et al. Gene expression analysis reveals matrilysin as a key regulator of pulmonary fibrosis in mice and humans. Proc Natl Acad Sci U S A. 2002;99(9):6292-6297.

25. Kurosu K, Takiguchi Y, Okada O, et al. Identification of annexin 1 as a novel autoantigen in acute exacerbation of idiopathic pulmonary fibrosis. J Immunol. 2008;181(1):756-767.

26. Borchers AT, Chang C, Keen CL, Gershwin ME. Idiopathic pulmonary fibrosis-an epidemiological and pathological review. Clin Rev Allergy Immunol. 2011;40(2):117-134.

27. Wallace WA, Ramage EA, Lamb D, Howie SE. A type 2 (Th2-like) pattern of immune response predominates in the pulmonary interstitium of patients with cryptogenic fibrosing alveolitis (CFA). Clin Exp Immunol. 1995;101(3):436-441.

28. Ohnishi H, Yokoyama A, Yasuhara Y, et al. Circulating KL-6 levels in patients with drug induced pneumonitis. Thorax. 2003;58(10): $872-875$.

29. Ritter M, Göggel R, Chaudhary N, et al. Elevated expression of TARC (CCL17) and MDC (CCL22) in models of cigarette smoke-induced pulmonary inflammation. Biochem Biophys Res Commun. 2005; 334(1):254-262. 


\section{Supplementary material}



Figure SI The schema of DILD screening using chest CT scan.

Abbreviation: DILD, drug-induced interstitial lung disease.

\section{Publish your work in this journal}

Therapeutics and Clinical Risk Management is an international, peerreviewed journal of clinical therapeutics and risk management, focusing on concise rapid reporting of clinical studies in all therapeutic areas, outcomes, safety, and programs for the effective, safe, and sustained use of medicines. This journal is indexed on PubMed Central, CAS,
EMBase, Scopus and the Elsevier Bibliographic databases. The manuscript management system is completely online and includes a very quick and fair peer-review system, which is all easy to use. Visit http://www.dovepress.com/testimonials.php to read real quotes from published authors.

Submit your manuscript here: http://www.dovepress.com/therapeutics-and-clinical-risk-management-journal 